\author{
Massimo Antonelli \\ Marc Bonten \\ Maurizio Cecconi \\ Jean Chastre \\ Giuseppe Citerio \\ Giorgio Conti \\ J. Randall Curtis \\ Goran Hedenstierna \\ Michael Joannidis \\ Duncan Macrae \\ Salvatore M. Maggiore \\ Jordi Mancebo \\ Alexandre Mebazaa \\ Jean-Charles Preiser \\ Patricia Rocco \\ Jean-François Timsit \\ Jan Wernerman \\ Haibo Zhang
}

Received: 13 December 2012

Accepted: 13 December 2012

Published online: 5 January 2013

(C) Springer-Verlag Berlin Heidelberg and ESICM 2013

As in previous years, Intensive Care Medicine published numerous articles dedicated to infection and sepsis, mostly focussing on pneumonia and related problems.

\section{Year in review in Intensive Care Medicine 2012. II: Pneumonia and infection, sepsis, coagulation, hemodynamics, cardiovascular and microcirculation, critical care organization, imaging, ethics and legal issues}

M. Cecconi

Anaesthesia and Intensive Care Medicine, St George's Hospital and Medical School, London, UK

\section{J. Chastre}

Reanimation Medicale, Hopital Pitié Salpétrière, Paris, France

\section{G. Citerio}

Neurointensive Care Unit, Ospedale S.

Gerardo, Monza, Italy

\section{J. R. Curtis}

Division of Pulmonary and Critical Care,

University of Washington, Seattle, USA

M. Antonelli $(\bowtie) \cdot G$. Conti •

S. M. Maggiore

Department of Intensive Care and Anesthesiology, Policlinico Universitario A. Gemelli, Università Cattolica del Sacro Cuore, Largo A. Gemelli, 8,

00168 Rome, Italy

e-mail: m.antonelli@rm.unicatt.it

Tel.: +39-06-30153226

\section{Bonten}

Department of Medical Microbiology, Julius Center for Health Sciences and Primary Care, University Medical Center Utrecht, Utrecht, The Netherlands

\section{G. Hedenstierna}

Department of Clinical Physiology,

Uppsala University, Uppsala, Sweden

M. Joannidis

Department of Internal Medicine, Medical University of Innsbruck, Innsbruck, Austria

\section{Macrae}

Pediatric Intensive Care Unit, Royal Brompton Hospital, London, UK

\section{J. Mancebo}

Intensive Care Medicine Unit, Hospital Sant

Pau, Barcelona, Spain
A. Mebazaa

Department of Anesthesiology and Critical Care Medicine, Lariboisière Hospital,

Paris, France

\section{J.-C. Preiser}

Department of Intensive Care, Erasme University Hospital, Brussels, Belgium

\section{P. Rocco}

Laboratory of Pulmonary Investigation, Centro de Ciências da Saúde, Rio de Janeiro, Brazil

\section{J.-F. Timsit}

Intensive Care Medicine Unit, Hôpital A. Michallon, Grenoble, France

\section{J. Wernerman}

Department of Anesthesiology and Intensive Care Medicine,

Karolinska University Hospital,

Stockholm, Sweden

\section{H. Zhang}

Interdepartmental Division of Critical Care Medicine, University of Toronto,

Toronto, Canada 


\section{Community-acquired pneumonia}

Different pathogens, variable virulence in different strains of microorganisms, increasing age, and underlying diseases undoubtedly have an important impact on community-acquired pneumonia (CAP) morbidity and mortality, but genetic variability affecting the host response may also influence susceptibility and outcome [1]. This is why Martin-Loeches and colleagues [2] assessed the genetic variability at IL6 in a large cohort of 1,227 white Spanish patients with CAP, of whom 306 had pneumococcal CAP (P-CAP). In patients with P-CAP multivariate analysis adjusted for age, gender, co-morbidity, hospital of origin, and severity showed that the ILC $-174 G G$ genotype was protective against the development of ARDS, septic shock, and multiple organ dysfunction syndrome. This effect was higher than that observed in patients with CAP, irrespective of the causal pathogen involved.

\section{Ventilator-associated pneumonia}

Whether azithromycin, a drug that could block the quorum-sensing (QS) system, could prevent ventilatorassociated pneumonia (VAP) in patients colonized by rhamnolipid-producing isolates is unknown. To test this hypothesis, Van Delden and colleagues [3] randomized 92 patients proven to be colonized by Pseudomonas aeruginosa, to receive either placebo or $300 \mathrm{mg} /$ day iv azithromycin in a double-blind fashion for a maximum of 20 days. Considering only the subgroup of patients at very high risk of developing VAP because they were colonized by isolates producing intermediate or high levels of rhamnolipids $(n=41)$, the incidence of VAP was reduced fivefold in azithromycin versus placebo patients $(1 / 5$ vs. $5 / 5 \mathrm{VAP}, p=0.048)$. These data stress the need for a large phase IIb clinical trial restricted to patients colonized by QS-proficient isolates to further establish safety and efficacy of QS inhibition by azithromycin to prevent $P$. aeruginosa VAP.

Fungal airway colonization is a frequent finding in patients submitted to mechanical ventilation (MV) and could promote VAP development, especially if caused by $P$. aeruginosa, a bacterium likely to become resistant to antimicrobial agents. Indeed, a growing body of evidence supports the strong interplay between Candida albicans and $P$. aeruginosa when coexisting within a biofilm environment such as the endotracheal tube or the airway $[4,5]$. In a prospective study of 323 consecutive cases of suspected VAP, Hamet et al. [6] were able to demonstrate that mortality rate was greater in patients with fungal airway colonization than in those without (44 vs. $31 \%$, $p=0.02$ ). Moreover, Candida spp. airway colonization was an independent risk factor for multidrug-resistant bacteria isolation, such as $P$. aeruginosa and Enterobacteriaceae [odds ratio (OR) $1.79,95 \%$ confidence interval (CI) $1.05-3.05 ; p=0.03]$, in addition to the time elapsed between ICU admission and VAP suspicion. Whether or not Candida spp. airway colonization should be considered when selecting an empiric antibiotic treatment for VAP will require additional studies, as well as for defining the clinical utility of antifungal drugs in such patients [7].

Etomidate has a favorable hemodynamic profile and is frequently used as a single bolus for rapid sequence intubation in trauma patients, as well as ICU patients. However, its use can induce transient corticosteroid insufficiency and thus increases susceptibility to nosocomial infection [8]. To investigate the impact of etomidate on the rate of hospital-acquired pneumonia (HAP) in trauma patients and the effects of hydrocortisone in etomidate-treated patients, Asehnoune and colleagues $[9,10]$ did a post hoc analysis of the HYPOLYTE double-blind, placebo-controlled trial of hydrocortisone in trauma patients. The administration of etomidate did not alter basal cortisolemia, but it did decrease the delta of cortisolemia at $60 \mathrm{~min}$, leading to corticosteroid insufficiency in $83 \%$ of the patients $(p=0.007)$. Forty-nine $(52 \%)$ patients with etomidate and $16(30 \%)$ patients without etomidate developed pneumonia by day 28 , respectively $(p=0.009)$. Among etomidate-exposed patients, hydrocortisone was found to significantly decrease the rate of HAP and the duration of MV. These data should alert physicians to limit the use of etomidate for the intubation of severe trauma patients unless they use hydrocortisone to counteract the deleterious effects of the drug.

Treatment of VAP is increasingly hampered by antibiotic resistance and an innovative approach is to augment antibiotic delivery at the site of infection.

Nebulized colistimethate sodium (CMS)/colistin is frequently used as an adjunctive therapy for the treatment of VAP in patients infected by very difficult-totreat pathogens, such as carbapenemase-producing Klebsiella pneumoniae or multiresistant strains of $P$. aeruginosa or Acinetobacter baumannii. However, very little information is available regarding the pharmacokinetics of the drug when used by aerosolization in ventilated patients. To fill this gap, Athanassa and colleagues [11] determined colistin concentrations in bronchoalveolar lavage fluid and serum of 20 patients mechanically ventilated, who had symptoms and signs suggestive of ventilator-associated tracheobronchitis caused by gram-negative bacilli only susceptible to polymyxin and who were treated by colistin inhalation at a dose of $1 \mathrm{MU}$ ( $80 \mathrm{mg}$ CMS) every $8 \mathrm{~h}$ via a vibrating-mesh nebulizer. Results demonstrated relatively high colistin concentrations in the epithelial lining fluid at 1 and $4 \mathrm{~h}$ and substantially lower concentrations at $8 \mathrm{~h}$ after inhalation, underlining that a 
dose of $80 \mathrm{mg}$ of inhaled CMS every $8 \mathrm{~h}$ may not be adequate for the treatment of patients with VAP due to multidrug-resistant gram-negative bacteria (GNB). Additional studies are required to determine whether larger doses of nebulized CMS may result in higher and sustained colistin concentrations at the target site in pulmonary infections.

A newly designed device for nebulizing amikacin (BAY41-6551) was used to treat 69 patients with VAP in a randomized, placebo-controlled, double-blind phase II study. Treatment was well tolerated and associated with good clinical cure rates that warrant further clinical evaluation [12].

Gram-negative bacteria predominate in HAP, and among them, Escherichia coli is the main Enterobacteriaceae involved in VAP. Phylogenetic analysis shows that E. coli population can be divided into four major groups (A, B1, B2, and D). Strains belonging to groups A and B1 are known to carry few genes encoding virulence factors (VF), with weak extraintestinal pathogenicity, and are commonly multidrug-resistant. On the other hand, group B2 and D strains typically express numerous VF mediating bacterial adhesion, cell host invasion, and toxicity, and usually exert high extraintestinal pathogenicity [13]. To provide a detailed analysis of $E$. coli strains colonizing and infecting ICU patients, Messika and colleagues [14] screened 132 consecutive ICU patients ventilated for longer than 3 days for simultaneous presence of E. coli in rectal, oropharyngeal, and respiratory samples (colonization or VAP). If present, $E$. coli isolates were characterized by antimicrobial susceptibility, phylogenetic grouping, and VF gene content. The authors found that multisite colonization with $E$. coli was frequent, observed in $19 \%$ of the patients. The majority of isolates belonged to phylogenetic group B2, characterized by high VF gene content and low antimicrobial resistance. Among screened VF genes, iron uptake genes such as iroN and sfa appeared important for lung infection. Targeting the associated proteins by way of vaccines or monoclonal antibodies may help establish novel therapeutic strategies in that setting.

\section{H1N1}

Pulmonary complications in the course of systemic infection might be clinically relevant. Although some isolated case reports of H1N1-associated rhabdomyolysis have been published, uncertainty still remains regarding the clinical implications of elevated creatine kinase (CK) levels in this setting [15]. To assess if increased CK was related to worse global, renal, and respiratory outcomes in critically ill patients with H1N1 infection, and if it could serve as a biomarker of severity, Borgatta and colleagues [16] retrospectively analyzed data gathered from 505 patients with confirmed H1N1 severe infection in Spain. They found that slight elevations were associated with increased pulmonary and kidney complications as well as increased length of stay (both ICU and hospital). Furthermore, starting from $\mathrm{CK}$ greater than $500 \mathrm{UI} / 1$, a breakpoint close to normal upper values, significant associations with worse renal outcomes were documented. These included higher occurrence of acute kidney injury (AKI), as well as more frequent need for renal replacement therapy, which progressively increased with rising $\mathrm{CK}$ values. Greater pulmonary injury was observed from CK greater than 1,000 UI/l, with $50 \%$ more involvement in chest radiograph in addition to more prolonged MV.

Early use of corticosteroids in patients affected by pandemic H1N1 influenza A infection, although relatively common, remains highly debated and might lead to opportunistic infections by Aspergillus fungi. Of 40 critically ill patients with confirmed H1N1 infection, 9 (23\%) developed invasive pulmonary aspergillosis (IPA) 3 days after ICU admission [17]. On multivariate analysis, use of corticosteroids before ICU admission was independently associated with IPA (OR 14.4, $p=0.007$ ), confirming that early use of corticosteroids in patients affected by pandemic H1N1 influenza A infection did not result in better outcomes and was associated with an increased risk of superinfection.

Since there are no guidelines for the design and conduct of randomized trials in pandemic critical illness, Annane et al. [18] presented their recommendations based on the experience they acquired during a multicenter randomized trial performed in ICU patients with 2009 H1N1 influenza pandemia. The authors report how they carried out a multicenter, randomized, placebo-controlled, double-blind trial of corticosteroids in ICU patients with 2009 H1N1 influenza pneumonia requiring MV. The feasibility of and hurdles in designing and initiating a phase III trial in a short-lived pandemic crisis were analyzed. The peak of the wave was missed by $2-3$ weeks and only 26 patients were randomized. The two main reasons for non-inclusion were patients' admission before the beginning of the trial and ICU personnel overwhelmed by clinical duties. Parallel rather than sequential regulatory and ethics approval, and preparation and masking of study drugs by local pharmacists would have allowed the study to start 1 month earlier. A dedicated research team in each participating center would have increased the ratio of screened to randomized patients. It was concluded that future pandemic research should consider an adaptive design. 


\section{Other infections and sepsis}

The spread of extended-spectrum beta-lactamase (ESBL)producing Enterobacteriaceae, notably $E$. coli and $K$. pneumoniae, to the community, and the need for prudent use of carbapenems require updated studies to identify current risk factors for colonization with ESBL-producing isolates in patients admitted to the ICU. To do that, Razazi and colleagues [19] conducted an 8-month prospective study in the medical ICU of an 850-bed general and university-affiliated hospital in France. ESBL-producing Enterobacteriaceae caused $3 \%$ of the infections diagnosed on admission. By multivariable analysis, transfer from another ICU, hospital admission in another country, surgery within the past year, prior neurologic disease, and prior administration of third-generation cephalosporin (within 3-12 months before ICU admission) were independent predictive factors of colonization by ESBL-producing isolates upon ICU admission. In carriers, ESBL-strains caused 10 and $27 \%$ of first and second episodes of ICU-acquired infections, respectively. These data may be useful for identifying which ICU patients really warrant empiric therapy with carbapenems for targeting ESBL-producing Enterobacteriaceae, and thus they may contribute to more efficient antibiotic stewardship programs to help control these organisms.

The recent increase in drug-resistant microorganisms complicates the management of hospital-acquired bloodstream infections (HA-BSIs). To investigate the epidemiology of HA-BSI and evaluate the impact of drug resistance on outcomes of ICU patients, controlling for patient characteristics and infection management, Tabah and colleagues [20] conducted a large, prospective, multicenter cohort study in 162 ICUs in 24 countries, enrolling a total of 1,156 patients who had developed such an infection. Overall, $48 \%$ of isolates were multidrugresistant, including $20.5 \%$ extensively resistant, and $0.4 \%$ pan-drug-resistant. The 28-day all-cause fatality rate was $36 \%$. Inadequate antibiotic treatment and failure to control the source of infection were both associated with 28-day mortality, independently from age, chronic co-morbidities, severity of acute illness, shock, and organ dysfunctions. Antimicrobial resistance was associated with a significantly longer time to adequate antimicrobial treatment and with a higher risk of death, even after controlling for adequacy of antimicrobial treatment. Intensified efforts to prevent HA-BSIs and to optimize their management through adequate source control and antibiotic therapy are clearly needed to improve outcomes.

Catheter-related infections (CRI) are an important source of HA-BSIs and represent relevant complications in ICU patients, although considerable achievements in infection prevention have been realized. In 51 French ICUs the overall CRI rate was 2.2 per 1,000 central venous catheter days in 7,188 patients and identified risk factors for CRI amenable for improvement were the preference of the subclavian route and the use of an antiseptic solution containing alcohol [21]. In a randomized study of 78 ICU patients with acute renal failure requiring hemodialysis, sodium citrate catheter locks were, as compared to saline locks, associated with less catheter complications, longer catheter life span, but comparable incidence of infections [22]. Another important infectious complication of treatment in ICU is the invasive candidiasis (IC). In a prospective study of 176 non-neutropenic patients with severe abdominal conditions at ICU admission and expected to stay at least 7 days, the value of $(1 \rightarrow 3)$ - $\beta$-D-glucan (BDG), C. albicans germ tube antibody (CAGTA), C-reactive protein (CRP), and procalcitonin (PCT) levels were assessed for the diagnosis of IC and for differentiating Candida spp. colonization from infection. BDG with a positive test for CAGTA accurately differentiated Candida colonization from IC with an area under the curve of 0.78 (95\% CI 0.76-0.81). CRP and PCT were less discriminatory [23].

Duration of the antibiotic treatment still remains a controversial problem. The European Society of Intensive Care Medicine (ESICM) working group performed a systematic review and meta-analysis of randomized clinical trials (RCTs) to determine whether plasma PCT determination can be used as a guide in order to shorten and optimize the duration of antibiotic therapy strategies for critically ill adult patients [24]. Seven evaluable RCTs were identified and analyzed. Primary outcomes included the duration of antibiotic therapy for the first episode of infection and 28-day mortality. Secondary outcomes included length of ICU stay, length of hospitalization, antibiotic-free days within the first 28 days of hospitalization, recurrences, and superinfections. The authors found that the duration of antibiotic therapy for the first episode of infection was reduced in favor of PCT-guided treatment [pooled weighted mean difference $(\mathrm{WMD})=-3.15$ days, random effects model, $95 \% \mathrm{CI}$ -4.36 to $-1.95, p<0.001]$. There was no difference in 28-day mortality between the compared arms [fixed effect model (FEM), OR 0.96, $95 \%$ CI 0.79-1.15, $p=0.63$ ). Antibiotic-free days were increased within the first 28 days of hospitalization in favor of the PCT-guided treatment arm (pooled WMD $=3.08$ days, FEM, $95 \%$ CI $2.06-4.10, p<0.001)$. No difference was found regarding the remaining outcomes. It was concluded that PCT-guided antibiotic therapy algorithms could help in reducing the duration of antimicrobial administration without having a negative impact on survival.

The increased number of infections caused by antimicrobial-resistant bacteria (AMRB) is increasing worldwide. Chlorhexidine body washing (CHG-BW) has been proposed as a measure to limit the spread of AMRB. Cross-transmission of AMRB is extremely important and temporarily contaminated hands of health-care workers 
are considered the most important vectors for spread. Derde et al. [25] systematically assessed the evidence on the effectiveness of CHG-BW in reducing colonization and infection with AMRB in adult ICU patients. Data from 16 studies were extracted; 9 were excluded because of assessed high risk of bias or inadequate analyses. The remaining studies differed markedly in interventions/cointerventions and case mix which precluded pooling of data in a formal meta-analysis, but overall the quality of the studies was good. It was found that the incidences of methicillin-resistant Staphylococcus aureus (MRSA) acquisition were reduced significantly in three studies in which this was the primary endpoint. A significant reduction in MRSA infection rates was observed in only one of five studies. The carriage and bacteremia rates of vancomycin-resistant enterococcus (VRE) were assessed in one study, and both significantly declined. Finally, there were hardly any data on the effects of CHG-BW on antibiotic-resistant gram-negative bacteria (ARGNB). It was concluded that CHG-BW may be effective in preventing carriage, and possibly BSIs, with MRSA and VRE in different ICU settings. As CHG-BW protocols, co-interventions, and case mix varied widely, the attribution of these effects to CHG-BW alone should be done with care. Evidence that CHG-BW reduces carriage of or infections with ARGNB is lacking.

Gut overgrowth happens in the critically ill requiring intensive care. Gut overgrowth is the crucial event which precedes both primary and secondary endogenous infection and is a risk factor for the development of de novo resistance. Selective decontamination of the digestive tract (SDD) is an antimicrobial prophylaxis designed to control overgrowth. In a review article Silvestri et al. [26] revised the mechanisms of action of SDD. Data assessed by the authors show that the full SDD regimen using parenteral and enteral antimicrobials reduces lower airway infection by $72 \%$, bloodstream infection by $37 \%$, and mortality by $29 \%$. Resistance is also controlled. Parenteral cefotaxime which reaches high salivary and biliary concentrations eradicates overgrowth of 'normal' bacteria such as $S$. aureus in the throat. Enteral polyenes control 'normal' Candida species. Enteral polymyxin and tobramycin eradicate or prevent gut overgrowth of 'abnormal' ARGNB. Enteral vancomycin controls overgrowth of 'abnormal' MSRA. SDD controls overgrowth by achieving high antimicrobial concentrations effective against 'normal' and 'abnormal' potential pathogens rather than by selectivity.

Early identification of infection and sepsis is crucial. Systemic levels of soluble urokinase-type plasminogen activator receptor (suPAR) positively correlate with the activation level of the immune system. Backes et al. [27] reviewed the usefulness of systemic levels of suPAR in the care of critically ill patients with sepsis, SIRS, and bacteremia, focusing on its diagnostic and prognostic value. Ten papers on original studies of critically ill patients were identified that report on suPAR in sepsis, SIRS, or bacteremia. Systematic levels of suPAR have little diagnostic value in critically ill patients with sepsis, SIRS, or bacteremia. Systemic levels of suPAR, however, have superior prognostic power over other commonly used biological markers in these patients. Mortality prediction by other biological markers or severity-of-disease classification system scores improves when combining them with suPAR. Systemic levels of suPAR correlate positively with markers of organ dysfunction and severity-of-disease classification system scores. Notably, the type of assay used to measure suPAR, the age of the patients, and underlying disease affect systemic levels of suPAR. The authors concluded that the diagnostic value of suPAR is low in patients with sepsis and infections and that the studies are limited to the predictive potential to estimate the mortality risk in observational designs.

The largest part of the sepsis burden occurs in middle- and low-income countries, and guidelines developed in high-income countries cannot be implemented in most middle- and low-income countries because lack of resources. Dunser et al. [28], representing a working group of the ESICM, provide a set of affordable recommendations for clinicians practicing in resourcelimited settings in order to improve the management of pediatric and adult septic patients. Recommendations are grouped into acute and post-acute interventions. Acute interventions include liberal fluid resuscitation to achieve adequate tissue perfusion, use of epinephrine or dopamine for inadequate tissue perfusion despite fluid resuscitation, measurement of arterial blood pressure in hemodynamically unstable patients, administration of hydrocortisone or prednisolone to patients requiring catecholamines, oxygen administration to achieve an oxygen saturation greater than $90 \%$, semi-recumbent and/or lateral position, non-invasive ventilation for increased work of breathing or hypoxemia despite oxygen therapy, administration of adequate antimicrobials, investigation for infectious source identification, fluid/ tissue sampling and microbiological work-up, removal, drainage, or debridement of the infectious source. Postacute interventions include regular re-assessment of antimicrobial therapy, administration of antimicrobials for an adequate but not prolonged duration, avoidance of hypoglycemia, pharmacological or mechanical deep vein thrombosis prophylaxis, resumption of oral food intake after resuscitation and regaining of consciousness, careful use of opioids and sedatives, early mobilization, and active weaning of invasive support. The authors concluded that scarce evidence exists for the management of pediatric and adult sepsis in resource-limited settings. These recommendations were endorsed by ESICM, the World Federation of Pediatric Intensive and Critical Care Societies (WFPICCS), the Global Sepsis Alliance (GSA), the Federation of Austrian Societies of Intensive 
Care Medicine (FASICM), the Ugandan Society of Intensive Care Medicine (USICM), and the Mongolian Society of Anesthesiology and Intensive Care Medicine (MSAICM).

Sepsis is a complex syndrome and adjunctive therapies to the conventional treatment might be of some benefit. Acetyl salicylic acid (ASA, aspirin) and non-steroidal anti-inflammatory drugs (NSAIDs) may have potential as adjunctive agents for sepsis. A review article [29] discussed the basis for sepsis therapy with ASA. ASA and NSAIDs have beneficial effects in numerous experimental models of sepsis. Low doses of ASA of $100 \mathrm{mg} / \mathrm{day}$ or less trigger synthesis of lipoxins, which are anti-inflammatory and aid in resolution of inflammation. Higher doses of ASA and NSAIDs act to reduce NF- $\kappa$ B stimulation and inhibit numerous septic pathways. While a previous randomized controlled trial of ibuprofen failed to show a reduction in mortality in sepsis, it did reduce clinical manifestations of sepsis. More recent observational studies have shown reduction in sepsis or acute lung injury leading to lower mortality in ICU patients treated with ASA. The author concluded that low-dose ASA appears to be beneficial in the prevention and treatment of sepsis and SIRS. If proven, this intervention would have a major, cost-effective impact on sepsis care.

Rare infections can be caused by criminal actions. A fraudulent use of Bacillus anthracis (anthrax) can be highly lethal. Two recent outbreaks related to contaminated mail in the USA and heroin in the UK and Europe and its potential as a bioterrorist weapon have greatly increased concerns over anthrax in the developed world. Hicks et al. [30] summarized the microbiology, pathogenesis, diagnosis, and management of anthrax. B. anthracis, a gram-positive bacterium, has typically been associated with three forms of infection: cutaneous, gastrointestinal, and inhalational. However, the anthrax outbreak among injection drug users has emphasized the importance of what is now considered a fourth disease form (i.e., injectional anthrax) that is characterized by severe soft tissue infection. While cutaneous anthrax is most common, its early stages are distinct and prompt appropriate treatment commonly produces a good outcome. However, early symptoms with the other three disease forms can be non-specific and mistaken for less lethal conditions. As a result, patients with gastrointestinal, inhalational, or injectional anthrax may have advanced infection at presentation that can be highly lethal. Once anthrax is suspected, the diagnosis can usually be made with gram stain and culture from blood or tissue followed by confirmatory testing (e.g., PCR). While antibiotics (ciprofloxacin/doxycycline/clindamycin) are the mainstay of anthrax treatment, use of adjunctive therapies such as anthrax toxin antagonists are a consideration. Prompt surgical therapy appears to be important for successful management of injectional anthrax.

\section{Coagulation and hemostasis}

Coagulation balances are considerably modified in severe ICU patients. Sepsis induces hypercoagulability, hypofibrinolysis, microthrombosis, and endothelial dysfunction leading to multiple organ failure. Recently, thrombin generation tests (TG) and point-of-care thromboelastography (TEG) or thromboelastometry emerged as dynamic global tests of hemostasis. Massion et al. [31] prospectively investigated analytical coagulation tests, TG assays, and thromboelastometric analyses from admission to day 7 in 29 patients with septic shock. The levels of most procoagulant factors were increased, as well as both delayed time and deficit in TG. Interestingly, prolonged aPTT $(p=0.007)$ and persistence of TG deficit $(p=0.024)$ on day 3 were independent predictors of mortality, independently from APACHE II score. The mechanisms of persistent coagulopathy as a potential new therapeutic avenue need further investigations.

Bleeding and thrombotic events are common in ICU. Bleeding events are common complications in patients with extracorporeal life support (ECLS) usually owing to mixed factors. Also, ECLS may promote high molecular weight multimers of Von Willebrand factor to uncoil and to be cleaved into less effective smaller multimers. It leads to acquired Von Willebrand syndrome (AVWS). In a prospective observational study, Heilmann and colleagues [32] confirmed that AVWS during ECLS was almost constant (31/32 patients) and associated with bleeding events in 22 cases. The relative contribution of AVWS and other coagulation disorders in bleeding events remained to be studied.

Even when standard of care subcutaneous prophylaxis is administrated, proximal deep venous thrombosis (DVT) rates in ICU patients may reach rates as high as $44 \%$. The thromboprophylaxis use in ICU does not follow international guidelines. In a survey performed in 59 Austrian ICUs, despite a median of four risk factors of DVT, the implementation of pharmacological and mechanical methods for thromboprophylaxis is only applied in $39 \%$ of patients, whereas $5 \%$ of the patients did not receive thromboprophylaxis at all. Current practice of thromboprophylaxis is predominantly based on the administration of low molecular weight heparins prescribed at rather arbitrary doses without a discernible relationship to drug monitoring, thromboembolic risk factors, vasopressor use, or fluid balance. The poor application of guidelines may reflect limited level of proof of the recommendation, or insufficient communication and physician knowledge of the guidelines [33].

Cheng et al. [34] confirmed that usual thromboprophylaxis is likely not adequate for ICU patients. Fifty patients from a surgical ICU having had major abdominal surgery for cancer were randomly allocated to either unfractionated heparin, subcutaneously three times daily 
(SQH) versus unfractionated heparin via intravenous infusion, titrated to an activated partial thromboplastin time of 40-45 s (IVH). Patients in the SQH group had no detectable peak anti-factor Xa (aFXa) activity for 5 days after surgery, whereas patients in the IVH group had statistically elevated levels compared to the SQH group on days 3-5. SQH patients demonstrated a hypercoagulable profile, whereas IVH patients displayed a normal profile. Current dosing schedules of unfractionated heparin for VTE prophylaxis may therefore be inappropriate in post-surgical ICU patients with cancer.

\section{Hemodynamic and cardiovascular}

Tissue oxygen saturation: prognostic values and effect of pressure and vasoconstriction

Guidelines on the hemodynamic management of patients with septic shock focus on macro hemodynamic parameters. Early manipulation of these variables, when coupled with treatment aiming at optimizing perfusion, namely monitoring central venous saturation $\left(\mathrm{ScVO}_{2}\right)$ or lactate clearance [35-37], has been associated with an improvement in outcome. Recently microcirculation was shown to provide additional information in the context of sepsis [38]; its assessment can provide extra information about prognostication in this population. Assessment of the microcirculation can be done with different devices. Near infrared spectroscopy (NIRS) technology has been used to assess tissue saturation in different clinical settings [38,39]. It is the dynamic assessment of changes in theStO${ }_{2}$ during a veno-occlusive test (VOT) that have demonstrated the most promising results in terms of prognostication in septic patients [40,41]. VOT slopes, evaluated as $\mathrm{StO}_{2}$ deoxygenation (DeOx) and the $\mathrm{StO}_{2}$ reoxygenation rate $(\mathrm{ReOx})$, can be affected by the mean arterial pressure (MAP), even when these changes occur during vasopressor therapy [42]. Whether the prognostic values of DeOx and ReOx are affected when the MAP has been restored after the resuscitation phase was not known. Mesquida et al. [43] tried to answer this question in a study in 33 patients with septic shock in which a MAP greater than $65 \mathrm{mmHg}$ had been restored with fluids and vasopressors. They looked at VOT slopes, MAP, sequential organ failure assessment (SOFA) scores, mortality, length of ICU, and length of hospital stay. They did find a strong correlation between the VOT slopes and MAP. Patients in which the SOFA score improved had more negative DeOx slopes. DeOx slope values were associated with no SOFA improvement with an OR of 1.48 (CI $95 \% 1.00-2.2, p=0.05$ ). Impairment in both $\mathrm{DeOx}$ and $\mathrm{ReOx}$ was associated with longer ICU and hospital stay. When correcting for MAP, DeOx was still associated with a worse outcome. In practice, while VOT slopes have prognostic implications, their values have to be interpreted in view of MAP value, too.

\section{Peripheral vasoconstriction influences thenar oxygen saturation as measured by NIRS}

Thenar $\mathrm{StO}_{2}$ and VOT slopes were studied by Lima et al. [44] to investigate the effect of peripheral vasoconstriction on the readings of thenar $\mathrm{StO}_{2}$ measured by NIRS. In an elegant study on healthy volunteers they explored whether peripheral vasoconstriction can affect the readings made by Inspectra model 650 (Hutchinson Technologies, Hutchinson, KS, USA), a device based on NIRS technology. Subjects were continuously monitored while actively cooled with a circulating cold-water blanket. The cooling process was controlled in order to avoid changes in the core temperature. Absolute values of $\mathrm{StO}_{2}$ and VOT slopes were recorded at different time intervals during the cooling and rewarming process. At the same time other measurements of macro-hemodynamics such as MAP, cardiac output (CO), and stroke volume (SV) were taken. During the controlled cooling process, MAP, $\mathrm{CO}$, and SV did not change significantly, whereas $\mathrm{StO}_{2}$ significantly decreased. There were also significant changes in the VOT re-oxygenation slope values associated with cooling. In practice, even without significant changes in systemic hemodynamic variables, peripheral vasoconstriction induced significant changes in the peripheral perfusion. This may have important implications when assessing thenar $\mathrm{StO}_{2}$ in critically ill patients. In fact, during the acute phase of shock, vasoconstriction is often present and it is therefore possible that observed changes in $\mathrm{StO}_{2}$ happen as a result of this $[45,46]$. The fact that SV, CO, and MAP did not change significantly during the cooling process is in line with other studies where changes in macro-hemodynamics did not correlate with changes in peripheral perfusion and measurements of $\mathrm{StO}_{2}$ [47]. Vascular tone can change often occur in critically ill patients and the results of this study suggest that peripheral vasoconstriction can markedly influence the readings of NIRS.

Knee area tissue oxygen saturation is predictive of 14-day mortality in septic shock. A group of investigators explored the prognostic value of NIRS in different areas of the body. Ait-Oufella et al. [48] studied whether $\mathrm{StO}_{2}$ measured around the knees can have a prognostic value in septic shock. The hypothesis was that, because mottling of the skin in patients with septic shock often tends to be more pronounced around the knees, then this area could be investigated in terms of prognostication of mortality and compared to a validated measurement such the thenar $\mathrm{StO}_{2}$. The authors compared knee $\mathrm{StO}_{2}$ and thenar $\mathrm{StO}_{2}$ at different time intervals during the stabilization phase of patients admitted with septic shock. They then looked at the association of 14-day mortality with knee $\mathrm{StO}_{2}$ and 
thenar $\mathrm{StO}_{2}$ during this evaluation. At $6 \mathrm{~h}$ after hemodynamic stabilization $\mathrm{knee} \mathrm{StO}_{2}$ was significantly lower in 14-day non-survivors compared to survivors. Thenar $\mathrm{StO}_{2}$ was also lower at 12,18 , and $24 \mathrm{~h}$ but less predictive than knee $\mathrm{StO}_{2}$. When looking at other variables associated with outcome at 14 days, $6 \mathrm{~h}$ arterial lactate, urinary output, and $\mathrm{ScVO}_{2}$ were also associated with mortality. After correcting for these factors, knee $\mathrm{StO}_{2}$ remained associated with mortality, showing it to be an independent predictor of mortality at 14 days in patients with septic shock.

Neutrophils: what is their role in the microcirculation?

Neutrophils are known to play a key role in sepsis. They represent a natural defense against bacterial infections, indeed neutropenia has been associated with increased susceptibility to infection. Despite this, activated leukocytes are also involved in pathophysiological processes that lead to organ injury [49]. For instance their pathological role has been demonstrated in mechanisms leading to endothelial dysfunction and during ischemia reperfusion injuries [50]. Confirmation to this theory comes also from experimental models where leukodepletion has been associated with a protective effect against reperfusion injuries [51]. The effect of neutropenia on the microcirculation in sepsis on humans was investigated in a study by Karvunidis et al. [52]. They studied the microcirculation via an orthogonal polarization spectral (OPS) imaging device in patients with septic shock, with and without neutropenia (defined as neutrophil count less than $\left.1 \times 10^{9} / 1\right)$, and in patients in which neutropenia was drug-induced. They also used a group of healthy volunteers as a control group. They did not find any difference in septic patients with or without neutropenia. Interestingly even in the chemotherapy-induced neutropenia group, there were similar alterations in the microcirculation. While neutrophil depletion has been hypothesized as a possible protective measure against sepsis-induced changes in the microcirculation, this study did not show any significant protective effect of neutropenia on the microcirculation, when assessing it with OPS examination.

Guytonian physiology at the bedside

The mean systemic filling pressure $\left(P_{\mathrm{msf}}\right)$ is the pressure anywhere in the circulation during circulatory arrest. According to the theory by Guyton [53] the venous return is a function of the gradient between the $P_{\mathrm{msf}}$ and the right atrial pressure. Venous return equals $\mathrm{CO}$, so estimating $P_{\text {msf }}$ at the bedside could represent an important functional measure of effective intravascular volume status. Maas et al. [54] compared three methods to estimate the
$P_{\mathrm{msf}}$ at the bedside. They used a previously validated method, namely the extrapolation for zero $\mathrm{CO}$ of central venous pressure (CVP)-CO pairs, during inspiratory hold maneuvers [55]; they also used a rapid arterial occlusion test $\left(P_{\text {arm }}\right)$ and an estimation of the $P_{\text {msa }}$ from a new device called a Navigator (Applied Physiology, Sydney, Australia). $P_{\text {arm }}$ assumes that during a rapid occlusion at a specific time point (after 25-30 s of stop flow) the arterial pressure equalizes the venous pressure, therefore allowing this test to estimate $P_{\mathrm{msf}}$. $P_{\mathrm{msa}}$ derives from a complex software algorithm that integrates CO, CVP, and MAP. $P_{\text {arm }}$ and $P_{\mathrm{msf}}$ showed very good agreement with minimal bias and narrow limits of agreement. During changes in the $P_{\mathrm{msf}}$ induced by either volume expansion or by a head tilt up both new methods, $P_{\mathrm{arm}}$ and $P_{\mathrm{msa}}$, tracked changes in the $P_{\mathrm{msf}}$ accurately.

Improving left ventricular relaxation with volume expansion in patients with septic shock

Diastolic dysfunction is not uncommon in patients with sepsis and septic shock [56]. Finding ways to improve ventricular relaxation represents an important part of therapy in these patients. To explore whether volume expansion, by improving SV and therefore myocardial perfusion, could have a role in improving relaxation, Mahjoub et al. [57] performed an echocardiographic study during rapid volume expansion in septic shock patients. They looked at the peak velocity of the mitral annulus early wave during diastole $\left(E^{\prime}\right)$ and the $E / E^{\prime}$ ratio (an index correlated to filling pressures such as the pulmonary artery occlusion pressure, PAOP) during rapid volume expansion. They found that in patients responding to fluids (defined as patients in which volume expansion produced an SV increase by more than $15 \%) E^{\prime}$ increased markedly only in responders with diastolic dysfunction, whereas it did not increase significantly in responders without diastolic dysfunction. This demonstrates that in patients with diastolic dysfunction responding to fluids, there is a concomitant improvement in relaxation. Interestingly, in non-responders there was a significant increase in the $E / E^{\prime}$ ratio, i.e., identifying an increase in filling pressure during volume loading. This index could possibly identify patients in which fluid expansion is not tolerated.

Completely non-invasive continuous blood pressure measurement in ICU

The ability to continuously monitor a physiological variable at the bedside is one of the cornerstones of intensive care medicine. A new technology, the T-line 200 (Tensys Medical, San Diego, CA, USA) based on arterial tonometry has recently become available. Saugel et al. [58] 
investigated the agreement of the T-line 200 with invasive arterial blood pressure in 28 intensive care patients. They found good bias $(+0.47 \mathrm{mmHg})$ comparing non-invasive MAP to invasive MAP, with limits of agreement between -16.53 and $+17.46 \mathrm{mmHg}$. For systolic arterial pressure (SAP), bias and limits of agreement were $-9.01(-37.47$ to 19.45$) \mathrm{mmHg}$ and for diastolic arterial pressure (DAP) they were $+5.22(-13.50$ to +23.94$) \mathrm{mmHg}$. These are interesting and promising results and they suggest that trending arterial blood pressure non-invasively via arterial tonometry may be a reality. More studies are needed in shocked patients, where often vasopressors are titrated against absolute values of MAP; at the moment such devices cannot be used as single blood pressure monitors.

\section{Fluid replacement therapies and fluid responsiveness}

This year in Intensive Care Medicine some papers and reviews analyzed the problem of fluid replacement therapies and indices of fluid responsiveness.

Response to fluid challenge is often defined as an increase in cardiac index (CI) of more than 10-15\%. However, in clinical practice CI values are often not available. In an observational study Pierrakos et al. [59] evaluated whether changes in MAP correlate with changes in CI after fluid challenge in patients with septic shock. The authors analyzed data prospectively collected from 51 septic shock patients, in whom complete hemodynamic measurements had been obtained before and after a fluid challenge with $1,000 \mathrm{ml}$ crystalloid (Hartman's solution) or $500 \mathrm{ml}$ colloid (hydroxyethyl starch (HES) 6). The authors divided the patients into two groups (responders and non-responders) according to their change in CI (responders: \% CI greater than $10 \%$ ) after the fluid challenge. The changes in MAP did not reliably track changes in CI after fluid challenge and the conclusion was that MAP should be interpreted carefully when evaluating the response to fluid challenge in septic shock patients.

In a study on the influence of the expiratory muscle activity on end-expiratory CVP, Leatherman et al. [60] showed that the changes in intra-abdominal pressure $(\triangle \mathrm{IAP})$ in 39 patients who had a respiratory excursion in CVP from end-expiration to end-inspiration (CVP(ee) CVP(ei)) of at least $8 \mathrm{mmHg}$, the CVP variations overestimated the transmural right atrial pressure. However the authors showed also that the magnitude of expiratory muscle activity can be assessed by measuring $\triangle \mathrm{IAP}$. Subtracting $\triangle \mathrm{IAP}$ from the end-expiratory $\mathrm{CVP}$ can provide a reasonable estimate of the CVP that would be obtained if exhalation were passive.

Hydroxyethyl starch is a synthetic colloid used widely for resuscitation despite the availability of safer, less costly fluids. Hartog et al. [61] investigated the potential reasons why HES use has become so widespread despite what appears to be objective data challenging its benefit and safety. They therefore examined the relationship between the methodological quality of published HES reviews, authors' potential conflicts of interest (pCOI), and the recommendations made. Between 1975 and 2010, 165 reviews were published containing recommendations for or against HES use. From the 1990s onwards, favorable reviews increased from two to eight per year and HES's share of the artificial colloid market tripled from 20 to $60 \%$. Methodology was discussed to minimize the ability of a select group of clinicians with undisclosed industry ties to flood the scientific literature with lowquality positive reviews and possibly affect sales of products that may potentially increase patient risks. This review stimulated an animated discussion.

Gelatin, a degradation product of collagen, is frequently used as a volume expander in critical care. Thomas-Rueddel et al. [62] aimed at investigating the current clinical evidence on the safety of this product and systematically reviewed the randomized controlled trials (RCTs) in patients receiving gelatin for resuscitation in comparison to albumin or crystalloids. A total of $40 \mathrm{RCTs}$ were published between 1976 and 2010 with 3,275 patients. Median sample size in the gelatin groups was 15 patients (range 10-249). Median gelatin dose was $17 \mathrm{ml} /$ $\mathrm{kg}$ (range 6-57 ml $/ \mathrm{kg})$. In 32 RCTs $(n=1,946 / 3,275$, $59 \%$ of all patients), the study period was $24.0 \mathrm{~h}$ at most. Twenty-nine RCTs $(n=2,001)$ investigated elective surgical patients, mostly undergoing cardiac surgery (18 RCTs, $n=819)$. Three RCTs $(n=723)$ investigated critically ill adults. Two RCTs $(n=59)$ were performed in emergency room patients, and six RCTs $(n=492)$ were performed in neonates or children. No study was adequately powered to investigate the frequency of patient-important outcomes. Risks were not statistically significantly different for mortality (RR 1.12, $95 \%$ CI $0.87-1.44$ ) and exposure to allogeneic transfusion (RR $1.28,0.89-1.83)$. Only three RCTs reported the occurrence of acute renal failure. It was concluded that despite over 60 years of clinical practice, the safety and efficacy of gelatin cannot be reliably assessed in at least some settings in which it is currently used.

Sandroni et al. [63] conducted a systematic review to assess the accuracy of the variation in pulse oximetry plethysmographic waveform amplitude $(\triangle \mathrm{POP})$ and the Pleth variability index (PVI) as predictors of fluid responsiveness in mechanically ventilated adults. Ten studies in 233 patients were included. All patients were in normal sinus rhythm. The pooled area under the receiver operating characteristic curve (AUC) for identification of fluid responders was 0.85 (95\% CI 0.79-0.92). Pooled sensitivity and specificity were 0.80 (95\% CI $0.74-0.85)$ and $0.76(0.68-0.82)$, respectively. No heterogeneity was found within studies with the same amount of fluid bolus, nor between studies on $\triangle \mathrm{POP}$ and those on PVI. The AUC was significantly larger in studies with a large bolus 
amount than in those with a small bolus $[0.92(95 \%$ CI $0.87-0.96)$ vs. $0.70(0.62-0.79) ; p<0.0001]$. Sensitivity and specificity were also higher in studies with a large bolus [0.84 (95\% CI $0.77-0.90)$ vs. $0.72(0.60-0.82)$ (small bolus), $p=0.08$ and 0.86 (95\% CI 0.75-0.93) vs. $0.68(0.56-0.77)$ (small bolus), $p=0.02]$, respectively. The authors concluded that on the basis of their metaanalysis, the $\triangle \mathrm{POP}$ and PVI are equally effective for predicting fluid responsiveness in ventilated adult patients in sinus rhythm. Prediction is more accurate when a large fluid bolus is administered.

Inotropes and vasoactive agents

Dobutamine is recommended for patients with severe heart failure and symptoms related to low $\mathrm{CO}$ and poor tissue perfusion. However there is concern and uncertainty regarding the harm-benefit balance associated with its use in this population. Tacon et al. [64] conducted this meta-analysis to evaluate whether dobutamine, compared with placebo or standard care, is associated with lower mortality and a range of secondary outcomes in patients with severe heart failure. Randomized trials comparing dobutamine with placebo or standard care, in human, adult patients with severe heart failure, were included if they reported at least one outcome of interest. Fourteen studies, with 673 participants, met the inclusion criteria and were included; 13 studies reported mortality. There was minimal heterogeneity $(I(2)=4.5 \%)$. The estimate of the OR for mortality for patients with severe heart failure treated with dobutamine compared with standard care or placebo was 1.47 (95\% CI 0.98-2.21, $p=0.06$ ). The authors concluded that dobutamine is not associated with improved mortality in patients with heart failure, and there is a suggestion of increased mortality associated with its use, although this did not reach the conventional level of statistical significance. It was also pointed out that studies had a poor level of methodological reporting.

Vasopressin infusion was shown to improve hemodynamic status in patients with vasodilatory shock, but may have relevant clinical side effects and its effect on mortality is controversial. Polito et al. [65] performed a meta-analysis to examine the effects of vasopressin or its analogue terlipressin on morbidity and mortality in patients with vasodilatory shock. The primary outcome for this meta-analysis was short-term all-cause mortality. Six studies were considered for the main analysis on mortality in adults. The crude short-term mortality was 206 of $512(40.2 \%)$ in vasopressin/terlipressin-treated patients and 198 of 461 (42.9\%) in controls [six trials, risk ratio $(\mathrm{RR})=0.91 ; 95 \%$ CI $0.79-1.05 ; p=0.21$; $I(2)=0 \%$ ]. There were 49 of $463(10.6 \%)$ patients with serious adverse events in the vasopressin/terlipressin arm and 51 of $431(11.8 \%)$ in the control arm (4 trials,
$\mathrm{RR}=0.90 ; 95 \%$ CI $0.49-1.67 ; p=0.75 ; I(2)=26 \%)$. It was concluded that the use of vasopressin or terlipressin did not produce any survival benefit in the short term in patients with vasodilatory shock.

Catecholamine vasopressor therapy in surgical ICU patients with cardiovascular failure may produce adverse cardiac events. Schmittinger et al. [66] investigated the occurrence of any of seven predefined adverse cardiac events (prolonged elevated heart rate, tachyarrhythmia, myocardial cell damage, acute cardiac arrest or death, pulmonary hypertension-induced right heart dysfunction, reduction of systemic blood flow) during catecholamine vasopressor therapy lasting at least $12 \mathrm{~h}$.

New-onset tachyarrhythmia (49.1\%), prolonged elevated heart rate $(23.7 \%)$, and myocardial cell damage $(17.5 \%)$ occurred most frequently. Globally, adverse cardiac events occurred in $44(48.2 \%)$ out of 112 of surgical ICU patients with cardiovascular failure and were related to morbidity and mortality. The extent and duration of catecholamine vasopressor therapy were independently associated with and may contribute to the pathogenesis of adverse cardiac events.

\section{Organization, quality of care, and outcome}

Medication errors are common in hospital. Opportunities for errors are maximized in ICUs. One simple way to limit errors in medication administration is to improve color-coded drug labelling. ISO norm 26825 for color drug labelling has been created in anesthesiology and extended for use in intensive care and emergency medicine. In a survey endorsed by ESICM, Balzer et al. [67] found that only $35 \%$ of the respondents reported that standardized drug labelling was used hospital-wide, and $39 \%$ reported that standardized drug syringe labelling (DSL) was used in their ICU (Europe: Northern $53 \%$, Western $52 \%$, Eastern $17 \%$, Southern $22 \%$ ). The ISO norm 26825 was used by $30 \%$, an adapted version by $19 \%$, and local versions by $45 \%$. The improvement of adherence to this simple norm should be an important tool for decreasing medication errors.

Variability of critical care bed numbers in Europe has been studied by Rhodes et al. [68]. Data were derived from large international databases and validated by the national council representative for the ESICM. In countries where data were still not available, personal contacts were used and numbers were estimated according to local sample assessment. They identified 409 acute care beds and 11.5 critical care beds on average per 100,000 inhabitants. The rates per 100,000 inhabitants were markedly different between countries: from 29.2 for Germany, 12.5 for Italy, 11.6 for France, 9.7 for Spain, 6 for Greece, to 4.2 for Portugal. The rates of critical care beds were correlated with percentage of gross domestic 
product dedicated to health expenditure with marked differences between countries.

Recent studies conducted on ICU patients have shown an advantage for patients treated in high volume centers and some have shown benefit with the adoption of centralized services for ICUs as well. Kanhere et al. [69] conducted a systematic review to assess if outcomes in adult ICUs are related to hospital and ICU patient volume. Twenty-five articles were relevant to this study. After further evaluation a total of 13 studies including 596,259 patients across 1,068 ICUs met the inclusion criteria and were reviewed. All were observational cohort studies. Four of the studies included all admissions to ICU, five included mechanically ventilated patients, two reported on patients admitted with sepsis, and one study each reported on patients admitted with medical diagnoses and post-cardiac arrest patients admitted to ICU, respectively. There was a wide variability in the quantitative definition of volume and classification of hospitals and ICUs on this basis. Methodological heterogeneity amongst the studies precluded a formal meta-analysis. A trend towards favorable outcomes for high volume centers was observed in all studies. Risk-adjusted mortality rates revealed a survival advantage for a specific group of patients in high volume centers in ten studies but no significant difference in outcomes was evident in three studies. It was concluded that outcomes of certain subsets of ICU patients (especially those on MV, high-risk patients, and patients with severe sepsis) are better in high volume centers within the constraints of risk adjustments.

Burnout syndrome (BOS) has frequently been reported in health-care workers, and precipitating factors include communication problems in the workplace and stress related to end-of-life situations. In a before-after study, Quenot et al. [70] evaluated the effect of an intensive communication strategy on BOS among caregivers working in ICU. The intervention was multifactorial, with mixed changes in ward organization (including unrestricted visiting hours, fixed appointments with families, staff psychologist availability) and in communication on BOS strategy. They found a significant difference between periods in all three components of the Maslach Burnout Inventory (MBI) (emotional exhaustion, $p=0.04$; depersonalization, $p=0.04$; personal accomplishment, $p=0.01$ ). MBI classified burnout as severe in $15(28 \%)$ caregivers in period 1 versus $7(14 \%)$ in period $2, p<0.01$, hence a $50 \%$ risk reduction. Symptoms of depression as evaluated by the CES-D were present in $9(17 \%)$ caregivers in period 1 versus $3(6 \%)$ in period $2, p<0.05$, hence a risk reduction of almost $60 \%$. Further randomized trials focused on interventions on communication factors are needed to confirm these results.
Outcome and quality of life after ICU

The age distribution of the population may contribute to the need for ICU beds to ensure the elderly beneficiate from ICU stay. In a retrospective study based on a database from the Beth Israel Diaconess Hospital in Boston, Fuchs et al. [71] evaluated the 28-day and 1-year impact of age while carefully taking into account comorbid condition and severity of the patients. Patients were divided into three age groups: 65-74, 75-84, and 85 and above. The analysis focused on the 7,265 non-planned admissions of patients above the age of 65 (45.7\% of the total ICU population). From the first to third age group there was an increased prevalence of cardiac comorbid illnesses, but a reduced prevalence of diabetes complications (7.5-2.4\%), alcohol abuse (4.1-0.6\%), metastatic cancer (8.3-3.6\%), chronic obstructive pulmonary disease (COPD) (24.4-17.4\%), and liver failure (5.0-1.0\%). Do not resuscitate order (DNRO) at admission increased from $6.8 \%$ in the 65-74 age group to $15.4 \%$ in the $75-84$ group and $29.6 \%$ in the 85 and over group $(p<0.001)$. Logistic regression analysis adjusted for gender, SOFA, DNRO, and Elixhauser comorbidity score found that patients from the second and third age group had ORs of 1.38 (95\% CI 1.19-1.59) and 1.53 (95\% CI 1.29-1.81) for 28-day mortality as compared with the first age group. Interestingly effect of age on the 1-year mortality was similar in the Cox regression analysis [75-84: adjusted hazard ratio 1.21 (95\% CI 1.06-1.38); 85 years and over: adjusted hazard ratio 1.59 (95\% CI 1.37-1.85)]. In conclusion, the effect of age adjusted for confounders was linear, although there was a non-significant modification in the slope of 1-year mortality over 84 years.

The impact of the precocity of ICU intervention in the prognosis of cancer patients is also an important matter of interest. In a retrospective monocenter study from Korea, Song et al. [72] studied the impact of an early intervention of a medical emergency team (MET) on the prognosis of cancer patients. They analyzed 199 critically ill cancer patients admitted to the ICU for in-hospital mortality. Median time from physiological derangement to intervention (time to intervention) prior to ICU admission was 1.5 (IQR 0.6-4.3) h. Median time to intervention was significantly shorter in survivors than in non-survivors (0.9 vs. $3.0 \mathrm{~h} ; p<0.001)$. Early intervention was associated with a lower SOFA score. Independent of the severity of illness, performance status, hematologic malignancy, stem-cell transplantation, presence of three or more abnormal physiological variables, presence of infection, need for $\mathrm{MV}$ and vasopressor, and low $\mathrm{PaO}_{2} /$ $\mathrm{FiO}_{2}$ ratio, the time from derangement to $\mathrm{ICU}$ admission was still significantly associated with hospital mortality 
(adjusted OR 1.45, $95 \%$ CI 1.22-1.72). The retrospective monocentric study argued for an early ICU admission of cancer patients. Further prospective study prospectively taking into account the reason for MET call, physician belief, and lead time bias (SOFA was measured only during MET intervention but not at the beginning of the acute care process) is warranted to confirm and quantify the effect.

Agitation and delirium are common in the critically ill. Nicotine withdrawal has been identified as a contributing factor to this. Nicotine replacement therapy may decrease withdrawal and improve ICU delirium and outcome. But nicotine replacement therapy was associated with an increased hospital mortality in a retrospective case control study likely owing to the sympathomimetic cardiovascular effect of nicotine. In a large retrospective cohort study of 423 smokers, after careful adjustment for confounders, and using various statistical models, Gillies et al. [73] did not find any association between NRT and hospital mortality. An RCT is therefore feasible to test the effect of NRT on delirium and patients' outcome.

Traumatic aortic injury is relatively infrequent but associated with a subsequent mortality if therapeutic management is delayed [74]. In a multicenter retrospective study involving 640 major trauma patients with associated blunt chest trauma, 76 were associated with aortic injury. They found that widened mediastinum, hypotension less than $90 \mathrm{mmHg}$, long bone fracture, pulmonary contusion, left scapula fracture, hemothorax, and pelvic fracture were independently associated with aortic injury. They proposed a risk score with an excellent accuracy (AUC ROC curve 0.96). External multicenter validation is needed to confirm this result.

It is important to decrease psychologic sequelae during recovery. In a multicenter RCT, McKinley and co-workers [75] studied the effect of physical rehabilitation on depression, anxiety, stress, and quality of sleep. The authors found that ICU survivors had impaired mental health and symptoms of depression, anxiety, stress, and psychological distress in the form of intrusive and avoidance post-traumatic stress symptoms. Anxiety symptoms persisted for one quarter of the patients after 8 and 26 weeks. Although physical rehabilitation did not decrease the psychological sequelae, they found that female gender, early levels of increased stress, and sleeping problems are associated with worse psychological recovery.

\section{Echography and imaging}

An expert panel conference report provided evidencebased consensus recommendations for lung ultrasound with a focus on emergency and critical care settings [76]. A multidisciplinary panel of 28 experts from eight countries was involved. The process used to develop these evidence-based recommendations involved two phases: determining the level of quality of evidence and developing the recommendation. The quality of evidence was assessed by the grading of recommendation, assessment, development, and evaluation (GRADE) method. However, the GRADE system does not enforce a specific method on how the panel should reach decisions during the consensus process. The methodology committee decided to utilize the RAND appropriateness method for panel judgment and decisions/consensus. Statements on pneumothorax, interstitial syndrome (pneumonia, atelectasis, pleural disease, etc.), monitoring of lung diseases (cardiogenic pulmonary edema, lung reaeration in ARDS, etc.), and neonatal and pediatric pulmonary problems were produced. It was concluded that the document was elaborated to guide implementation, development, and training on the use of lung ultrasound in all relevant settings.

Another group of experts provided recommendations on ultrasound guidance during vascular access [77]. Also these authors used the GRADE and the GRADE-RAND methods to develop recommendations. The recommendations suggest the advantage of $2 \mathrm{D}$ vascular screening prior to cannulation and that real-time ultrasound needle guidance with an in-plane/long-axis technique optimizes the probability of needle placement. Ultrasound guidance can be used not only for central venous cannulation but also in peripheral and arterial cannulation. Ultrasound can be used in order to check for immediate and life-threatening complications as well as the catheter's tip position. Educational courses and training are required to achieve competence and minimal skills when cannulation is performed with ultrasound guidance. This technique allows the reduction of infectious and mechanical complications. A recommendation to create an ultrasound curriculum on vascular access was proposed. It was concluded that given the evidence from literature and experts' opinion, ultrasound guidance has to be suggested as the method of choice for any kind of vascular cannulation given its higher safety and efficacy.

A review article discusses technical requirements for Doppler assessment of the small intra-renal vessels, factors influencing Doppler-based renal resistive index (RI), and to provide an updated description of its interest with a special focus on the critically ill [78]. Data suggest that renal Doppler may help to assess perfusion of native or transplanted kidneys, and recent studies suggest that it may be useful in critically ill patients. Renal Doppler has proven to be valuable for assessing large arterial or venous abnormalities and has been suggested to assess renal perfusion and to predict AKI in critically ill patients. However, numerous factors have been shown to influence renal Doppler and may constitute potential confounding factors. It was concluded that Doppler-based RI seems to be a promising tool in the critically ill to assess the risk of AKI, help in differentiating persistent from transient AKI, 
or assess changes in renal perfusion as consequences of therapeutic intervention. The authors also pointed out that the role of renal Doppler as a monitoring tool in the ICU will remain unclear until additional studies are conducted and the potential confounding factors are properly assessed.

Lakhal et al. [79] analyzed the current practices of chest radiograph (CXR) prescription and their clinical impact in French ICUs with a prospective snapshot observational study (on RadioDay) combined with a survey. On RadioDay, 854 CXRs (in 804 patients) were ordered. For the "CXRs morning round", the prescription policy was declared to be "on-demand" (in $63 \%$ of the ICUs), "daily routine only in mechanically ventilated patients (MV)" (30\%) or, less frequently, "daily routine in all patients" (7 \%). When analyzing the two main local policies, as compared with "daily routine only in MV" ICUs, in "on-demand" ICUs: (1) fewer CXRs were ordered $\quad(0.6 \pm 0.3 \quad$ vs. $0.9 \pm 0.2 \quad \mathrm{CXRs} /$ patient, $p<0.001)$ with no increase in the rate of unscheduled CXRs (i.e., CXRs performed outside the morning round), and (2) individual CXRs were more often followed by a therapeutic intervention (which would not have occurred without the CXR): 34 vs. $25 \%$ of the CXRs $(p<0.05)$. Nearly two-thirds of the participating ICUs adopted the "on-demand" strategy of prescription, which was associated with a lower rate of CXRs with no increase in unscheduled CXRs and was of higher clinical value than a "daily routine in MV" strategy.

Rohacek et al. [80] performed a prospective survey among the emergency physicians on the reason for ordering a CT pulmonary angiography (CTPA) in the emergency room. The most frequent reasons for ordering a CTPA were to confirm/rule out pulmonary embolism (PE) $(93 \%)$, elevated D-dimers $(66 \%)$, fear of missing PE (55\%), and Wells/simplified revised Geneva score (53\%). Interestingly, a positive answer for "fear of missing PE" was inversely associated with positive CTPA (OR 0.36, $95 \%$ CI 0.14-0.92, $p=0.033$ ), and "Wells/simplified revised Geneva score" was associated with positive CTPA (OR 3.28, $95 \%$ CI 1.24-8.68, $p=0.017)$. The proportion of positive CTPA was higher if a questionnaire was completed (26.4 vs. $14.5 \%$, OR $2.12,95 \%$ CI $1.36-3.29, p<0.001)$.

\section{Ethics and legal section}

In the past year, Intensive Care Medicine has published a number of important articles in the "Ethics and Legal Section" that have addressed a diverse group of topics including the ethics of clinical research in the ICU, the provision of end-of-life care in the ICU, and the impact of intensive care on the emotional and psychological health of ICU clinicians.
Ethics of clinical research in end-of-life care

There has been increasing interest in examining the ethics of conduct of clinical research in the ICU. Critically ill patients and their family members represent a vulnerable population for research and it is important that we protect this vulnerable group. At the same time, delivery of highquality care requires clinical research to ensure that we understand which therapies and approaches are most effective and that we implement these therapies and approaches in an efficient and timely way. Barrett and colleagues [81] performed a cross-sectional study of the attitudes of surrogate decision-makers toward research decision-making for critically ill patients. They presented 136 surrogate decision-makers of critically ill patients in five ICUs in Canada with four different hypothetical research scenarios during a structured interview. The four research scenarios included a placebo-controlled RCT, a study with higher risk of treatment complication, a study comparing two accepted treatments, and a study with a short enrolment window. They found that $90 \%$ of participants wished to be involved in research decisionmaking and participants' responses varied little across the different hypothetical studies. Eighty-five percent of participants considered their involvement in the consent process to be acceptable, but the proportion decreased when risk of harm was higher or the enrolment window was shorter. These authors did not find an association between attitudes toward participation in research and levels of symptoms of anxiety and depression. Specific suggestions to improve the consent process endorsed by the majority surrogate decision-makers included having the consent process preceded by a medical update from the ICU physician, having the ICU physician involved in the research decision-making process, having a discussion about the research in a private setting, and providing more verbal information than is generally given.

A single-center study from Canada by Mehta and colleagues [82] used questionnaires to explore the reasons that surrogate decision-makers agreed or declined to participate in one of a series of studies concerning critically ill patients. The questionnaires explored surrogate decision-makers' opinions about research in general, timing of research approach, the informed consent process, and reasons for agreeing or declining participation. The investigators enrolled 96 surrogate decision-makers including 68 who agreed to participation in an ICU study and 27 who declined participation. There were no differences between the two groups with respect to demographics, perceived severity of illness of the patient, or the research study approach. The most common reasons for providing consent were potential for research to help others, research being important for medical progress, and trust in the medical team. The most common reasons for declining consent were that the surrogate decision-maker was too anxious to consider research, fear 
that the patient would receive experimental treatment, and concern about risks of the study. This study also suggests that having two or more family members involved in the consent process may help family members with the stress of considering research and could enhance research participation.

Menon and colleagues examined consent rates in pediatric critical care research and the association between consent rates and multiple factors including patient, legal guardian, consent process, and study designrelated factors. They conducted a prospective cohort study in six pediatric ICUs in Canada with legal guardians of patients who were approached for consent for any research study. They recorded 271 consent encounters. The overall consent rate was $80 \%$ and they observed higher consent rates when research assistants were introduced to the family by a member of the clinical team. Interestingly, there was no difference in consent rates between therapeutic versus non-therapeutic studies. This study suggests researchers should consider routine introduction of the research assistant to the family by a member of the patient's care team.

Influence of physician attitudes and education and of ICU culture on end-of-life decision-making

There were three interesting articles in 2012 that examined the role of physicians and ICU culture on end-of-life decision-making. Schenker and colleagues [83] examined the association between physicians' beliefs and the presentation to the family of the option of comfort care for critically ill patients. This was a mixed-methods study of 72 audio-recorded family conferences about treatment decisions in ICUs at two hospitals in San Francisco, CA. The family conferences concerned patients at high risk of death or severe functional impairment and $72 \%$ died during this hospitalization. A total of 169 family members and 54 physicians participated. The authors examined the audio-recorded conferences to identify whether physicians offered comfort care as an alternative to lifesustaining treatment. Physicians completed a questionnaire indicating the strength of their belief that life support should be foregone. Using a broad definition of comfort-oriented treatment, Schenker and colleagues found that this option was presented in $56 \%$ of conferences. The only independent predictor of offering comfort care as an option was the strength of the physician's belief that life support should be foregone, suggesting that a physician's belief that the appropriate goal of care is life prolongation may result in inappropriately withholding the option of comfort care from some patients and their family members.

A study by Forte and colleagues [84] from Brazil examined the association between physician education in end-of-life care and variability in clinical practice. The authors surveyed physicians from 11 ICUs at a university hospital concerning a hypothetical patient in a vegetative state with no family or advance directives. They obtained a response rate was $89 \%$, with 105 questionnaires analyzed. They found that physicians who did not apply DNROs were less likely to have attended classes on end-of-life care. They found that physicians who would apply "full code" to the hypothetical patient had less often read about end-of-life care and had less interest in discussing end-of-life care with colleagues. These results demonstrate that education in endof-life care is associated with physician self-reported practice patterns regarding end-of-life care.

A unique study by Barnato and colleagues [85] examined the norms of decision-making in the ICUs of two academic medical centers in one city that represented the two extremes in intensity of care at the end-of-life. The authors conducted a 4-week mixed-methods case study at each medical center involving direct observation of patient care during rounds in the main medical ICU, semi-structured interviews with staff, patients, and families, and collection of materials such as patient lists and standardized forms. They compared patterns of decision-making regarding initiation, continuation, and withdrawal of life support using qualitative research methods. They observed 80 patients cared for by 4 senior physicians at the lowintensity medical center and observed 73 patients cared for by 4 senior physicians at the high-intensity medical center. Life support initiation was similar, but the low-intensity medical center was more likely to use a time-limited trial of life support, followed by withdrawal of life support. They also identified important differences between the two medical centers in the ultimate goals of life support, the determination of when a patient is considered to be "dying", a focus on concerns about harms of commission versus omission, and physician self-efficacy for life support decision-making. This study highlights some of the differences in the medical culture between ICUs that provide highintensity care at the end of life compared to those that provide low-intensity care at the end of life.

\section{Provision of end-of-life care in the ICU}

There were two interesting studies about the provision of end-of-life care in the ICU. In the first, Azoulay and colleagues [86] examined whether patients who had a birthday during their ICU stay seemed to a receive different level of care than those who did not. They conducted a case-control design in which patients spending their birthday in the ICU (cases) were matched to controls in terms of hospital, gender, age, severity of illness, and type of admission. The study was conducted in 12 ICUs in French hospitals. They found that compared with controls, the cases were more often trauma patients and received a larger number and longer durations of lifesustaining interventions. This increased intensity of life 
support occurred after, but not before, the birthday. The cases had longer ICU stays, although ICU and hospital mortality were not different between the two groups. Decisions to limit life support were made in the same proportion in both groups (22 and $24 \%$ of cases and controls, respectively), but these decisions were made significantly later in the cases than in the controls. Although the reasons for this association are not clear, the study suggests that patients who spent their birthday in the ICU were more likely to receive non-beneficial interventions.

In the second study, Bulow and colleagues [87] examined differences in decisions about end-of-life care and respect for patient autonomy across different religions and religiosity. They examined results of structured questionnaires regarding decisions about end-of-life care that were completed in six European countries in 142 ICUs. This study focused on responses from Protestants, Catholics, and Jews, the largest categories of religions in the sample. They examined a total of 304 physicians, 386 nurses, 248 patients, and 330 family members, finding that professionals wanted less treatment for themselves (ICU admission, CPR, ventilator treatment) than did patients and family members. Respondents reporting higher religiosity (defined by self-report as "religious" or "very religious") wanted more treatment and were more in favor of life prolongation. There were no differences between religions and religiosity regarding patient's autonomy. However, Protestants were more likely to report the importance of following competent patients' wishes and the Jewish respondents least likely to do so. Jewish professionals more frequently accepting patients' wishes for futile treatment. This study adds to the literature that examines the influence of religion and religiosity on patient, family, and health-care professionals' attitudes toward end-of-life care.

\section{Regional studies of end-of-life care in the ICU}

There were several studies that examined end-of-life care or triage decisions in different countries. In a study from France, Quenot and colleagues [88] examined the impact of a communication strategy on end-of-life care in the ICU after passage of a 2005 French law pertaining to the family's role in decision-making for critically ill patients. The authors examined whether an intensive communication strategy based on this law would influence practices in terms of withholding and withdrawing treatment and patient outcome. They conducted a single-center, twoperiod study performed before and after the 2005 French law (a law specifying new patient and family member rights regarding end-of-life care) using data obtained from the medical record. Between these periods, an intensive strategy for communication was developed and implemented, comprising regular meetings and specific changes in the organization of the ICU with regard to family communication and approaches to communication with families (such as increased family visiting hours). Of 2,478 patients admitted in period $1,27 \%$ died in the ICU compared to $28 \%$ in period 2 . In period 1, among patients who died in the ICU, $45 \%$ died subsequent to a decision to withhold or withdraw life support versus a significantly higher $85 \%$ in period 2 . The median time delay between ICU admission and initiation of decisionmaking process was significantly shorter in period 2 (6-7 vs. 3-5 days). Similarly, median time from admission to a decision to withhold or withdraw life support was significantly shorter in period 2 (11-13 vs. 4-6 days). In addition, median time from admission to death in the ICU after a decision to withhold or withdraw life support was significantly shorter in period 2 . The reasons documented for withholding or withdrawing life support were not significantly different between periods. This study suggests that an intensive communication in combination with a new law increasing family members' role in decision-making was associated with faster decisionmaking about end-of-life care in the ICU.

There were also two studies examining ICU care in developing countries, about which there has been little research. In the first, Ouanes and colleagues [89] examined decisions about end-of-life care in two Tunisian ICUs. The authors examined consecutive deaths that occurred in a medical and a surgical ICU in one hospital over 2 years. Decisions about end-of-life decisions were prospectively recorded by the senior attending physicians. They found that 326 of 1,733 patients died (19\%). A decision for full support was made in $69 \%$, whereas decisions to withhold or withdraw life support were made in 22 and $9 \%$ of deaths, respectively. The decisions about end-of-life care were similar in the medical and surgical ICUs. There was no instance of withdrawal of MV. Of the 20 patients who died after withdrawal of life support in the medical ICU, 10 patients were discharged to die at home. Two factors were independently associated with decisions to withhold or withdraw life support: a severe and ultimately fatal underlying disease and the absence of independent functional status before the ICU. The authors conclude that withholding and withdrawing life support are common in medical and surgical ICUs of a Tunisian hospital.

In the second, Louriz and colleagues [90] examined the determinants and outcomes associated with decisions to deny or delay ICU admission to a medical ICU in Morocco. Over a 6-month period, ICU admission was requested for 398 patients: $28 \%$ were immediately admitted, $36 \%$ were never admitted, and $36 \%$ were admitted at a later time. The reasons for refusal included too sick to benefit (11\%), too well to benefit (19\%), unit full $(41 \%)$, and more patient data needed to make a decision $(30 \%)$. Predictors of ICU refusal for neveradmitted patients included severity of acute illness and lack of available ICU beds. Hospital mortality was lowest 
for immediately admitted patients at $33 \%$ and higher for patients admitted later $(44 \%)$ and for never-admitted patients $(49 \%)$.

Gristina and colleagues [91] published a commentary addressing the limitations of a proposed new Italian regulation concerning advance directives. In Italy, the role of advance directives has recently been the subject of debate involving political parties and the Roman Catholic Church. In February 2009, a government coalition presented a bill of law on this issue that has been passed by the Lower Chamber. The authors' goal in this commentary is to highlight the bill's contradiction with Italian Constitution, Italian Code of Medical Ethics (ICME), and Oviedo Convention contents through an analysis of the bill's content in the light of these articles and by comparison with French legislation regarding end of life (Leonetti law). The authors conclude that advance directives should represent informed preferences made freely by patients within the relationship with their physicians, as part of an advance care planning. They further conclude that the law should be revised to meet ICME principles, assigning advance directives a juridical power, acknowledging their validity in legitimating end-of-life decisions, and defining a framework of juridical legitimacy for these decisions without infringing on patients' right to autonomy regarding health care.

Role of patient's socioeconomic status, and predictors of resilience in ICU clinicians

Two additional studies examined interesting aspects of the delivery of intensive care. Few studies have examined the role of socioeconomic status (SES) on care for critically ill patients, especially focusing on the surgical ICU.
Bein and colleagues [92] examined whether SES was associated with the severity of illness at admission, length of stay, and level of family member involvement in an adult surgical ICU. This was a cohort study of 1,006 patients at a 24-bed surgical ICU in Germany. They found that patients with low SES had higher severity of illness, defined by the SOFA score, at admission as compared with patients with high SES. When compared with men with high SES, those with low SES were more likely to receive ICU treatment for at least 5 days and showed a trend for a lower number of visits from family. Similar trends were not identified in women.

Mealer and colleagues [93] conducted a qualitative study of resilience and post-traumatic stress disorder (PTSD) among ICU nurses in the USA. The goal of the study was to identify mechanisms employed by highly resilient ICU nurses to provide insights into potential preventative therapies to obviate the development of PTSD in ICU nurse. They conducted a qualitative study using semi-structured telephone interviews with randomly selected ICU nurses in the USA. Purposive sampling was used to identify ICU nurses who were highly resilient based on a validated scale of resilience and those whose score on a validated post-traumatic diagnostic scale was consistent with a diagnosis of PTSD. The authors used a constructivist epistemological framework for data analysis. Highly resilient nurses identified spirituality, a supportive social network, optimism, and having a resilient role model as characteristics used to cope with stress in their work environment. ICU nurses with a diagnosis of PTSD possessed several unhealthy characteristics including a poor social network, lack of identification with a role model, disruptive thoughts, regret, and lost optimism. These characteristics and skills may be used to develop target therapies to prevent PTSD in ICU nurses.

\section{References}

1. Jones SA (2005) Directing transition from innate to acquired immunity: defining a role for IL-6. J Immunol 175:3463-3468

2. Martin-Loeches I, Sole-Violan J, Rodriguez de Castro F, Garcia-Laorden MI, Borderias L, Blanquer J, Rajas O, Briones ML, Aspa J, Herrera-Ramos E, Marcos-Ramos JA, Sologuren I, Gonzalez-Quevedo N, Ferrer-Aguero JM, Noda J, Rodriguez-Gallego C (2012) Variants at the promoter of the interleukin-6 gene are associated with severity and outcome of pneumococcal community-acquired pneumonia. Intensive Care Med 38:256-262
3. van Delden C, Kohler T, BrunnerFerber F, Francois B, Carlet J, Pechere JC (2012) Azithromycin to prevent Pseudomonas aeruginosa ventilatorassociated pneumonia by inhibition of quorum sensing: a randomized controlled trial. Intensive Care Med 38:1118-1125

4. Azoulay E, Timsit JF, Tafflet M, de Lassence A, Darmon M, Zahar JR, Adrie C, Garrouste-Orgeas M, Cohen Y, Mourvillier B, Schlemmer B (2006) Candida colonization of the respiratory tract and subsequent pseudomonas ventilator-associated pneumonia. Chest 129:110-117
5. Roux D, Gaudry S, Dreyfuss D, ElBenna J, de Prost N, Denamur E, Saumon G, Ricard JD (2009) Candida albicans impairs macrophage function and facilitates Pseudomonas aeruginosa pneumonia in rat. Crit Care Med 37:1062-1067

6. Hamet M, Pavon A, Dalle F, Pechinot A, Prin S, Quenot JP, Charles PE (2012) Candida spp. airway colonization could promote antibiotic-resistant bacteria selection in patients with suspected ventilator-associated pneumonia. Intensive Care Med 38:1272-1279

7. Ricard JD, Roux D (2012) Candida colonization in ventilated ICU patients: no longer a bystander! Intensive Care Med 38:1243-1245 
8. Jabre P, Combes X, Lapostolle F, Dhaouadi M, Ricard-Hibon A, Vivien B, Bertrand L, Beltramini A, Gamand P, Albizzati S, Perdrizet D, Lebail G, Chollet-Xemard C, Maxime V, BrunBuisson C, Lefrant JY, Bollaert PE, Megarbane B, Ricard JD, Anguel N, Vicaut E, Adnet F (2009) Etomidate versus ketamine for rapid sequence intubation in acutely ill patients: a multicentre randomised controlled trial. Lancet 374:293-300

9. Asehnoune K, Mahe PJ, Seguin P, Jaber S, Jung B, Guitton C, Chatel-Josse N, Subileau A, Tellier AC, Masson F, Renard B, Malledant Y, Lejus C, Volteau C, Sebille V, Roquilly A (2012) Etomidate increases susceptibility to pneumonia in trauma patients. Intensive Care Med 38:1673-1682

10. Roquilly A, Mahe PJ, Seguin P, Guitton C, Floch H, Tellier AC, Merson L, Renard B, Malledant Y, Flet L, Sebille V, Volteau C, Masson D, Nguyen JM, Lejus C, Asehnoune K (2011) Hydrocortisone therapy for patients with multiple trauma: the randomized controlled HYPOLYTE study. JAMA 305:1201-1209

11. Athanassa ZE, Markantonis SL, Fousteri MZ, Myrianthefs PM, Boutzouka EG, Tsakris A, Baltopoulos GJ (2012) Pharmacokinetics of inhaled colistimethate sodium (CMS) in mechanically ventilated critically ill patients. Intensive Care Med 38:1779-1786

12. Niederman MS, Chastre J, Corkery K, Fink JB, Luyt CE, Garcia MS (2012) BAY41-6551 achieves bactericidal tracheal aspirate amikacin concentrations in mechanically ventilated patients with Gram-negative pneumonia. Intensive Care Med 38:263-271

13. Croxen MA, Finlay BB (2010) Molecular mechanisms of Escherichia coli pathogenicity. Nat Rev Microbiol 8:26-38

14. Messika J, Magdoud F, Clermont O, Margetis D, Gaudry S, Roux D, Branger C, Dreyfuss D, Denamur E, Ricard JD (2012) Pathophysiology of Escherichia coli ventilator-associated pneumonia: implication of highly virulent extraintestinal pathogenic strains. Intensive Care Med 38:2007-2016

15. Ayala E, Kagawa FT, Wehner JH, Tam J, Upadhyay D (2009) Rhabdomyolysis associated with 2009 influenza A(H1N1). JAMA 302:1863-1864
16. Borgatta B, Perez M, Rello J, Vidaur L, Lorente L, Socias L, Pozo JC, Pozo J, Garnacho-Montero J (2012) Elevation of creatine kinase is associated with worse outcomes in 2009 pH1N1 influenza A infection. Intensive Care Med 38:1152-1161

17. Wauters J, Baar I, Meersseman P, Meersseman W, Dams K, De Paep R, Lagrou K, Wilmer A, Jorens P, Hermans G (2012) Invasive pulmonary aspergillosis is a frequent complication of critically ill H1N1 patients: a retrospective study. Intensive Care Med 38:1761-1768

18. Annane D, Antona M, Lehmann B, Kedzia C, Chevret S (2012) Designing and conducting a randomized trial for pandemic critical illness: the 2009 H1N1 influenza pandemic. Intensive Care Med 38:29-39

19. Razazi K, Derde LP, Verachten M, Legrand P, Lesprit P, Brun-Buisson C (2012) Clinical impact and risk factors for colonization with extendedspectrum beta-lactamase-producing bacteria in the intensive care unit. Intensive Care Med 38:1769-1778

20. Tabah A, Koulenti D, Laupland K, Misset B, Valles J, Bruzzi de Carvalho F, Paiva JA, Cakar N, Ma X, Eggimann $\mathrm{P}$, Antonelli M, Bonten MJ, Csomos A, Krueger WA, Mikstacki A, Lipman J, Depuydt P, Vesin A, Garrouste-Orgeas M, Zahar JR, Blot S, Carlet J, BrunBuisson C, Martin C, Rello J, Dimopoulos G, Timsit JF (2012) Characteristics and determinants of outcome of hospital-acquired bloodstream infections in intensive care units: the EUROBACT International Cohort Study. Intensive Care Med 38:1930-1945

21. Timsit JF, L'Heriteau F, Lepape A, Francais A, Ruckly S, Venier AG, Jarno P, Boussat S, Coignard B, Savey A (2012) A multicentre analysis of catheter-related infection based on a hierarchical model. Intensive Care Med 38:1662-1672

22. Hermite L, Quenot JP, Nadji A, Barbar SD, Charles PE, Hamet M, Jacquiot N, Ghiringhelli F, Freysz M (2012) Sodium citrate versus saline catheter locks for non-tunneled hemodialysis central venous catheters in critically ill adults: a randomized controlled trial. Intensive Care Med 38:279-285
23. Leon C, Ruiz-Santana S, Saavedra P, Castro C, Ubeda A, Loza A, MartinMazuelos E, Blanco A, Jerez V, Ballus J, Alvarez-Rocha L, Utande-Vazquez A, Farinas O (2012) Value of beta-Dglucan and Candida albicans germ tube antibody for discriminating between Candida colonization and invasive candidiasis in patients with severe abdominal conditions. Intensive Care Med 38:1315-1325

24. Matthaiou DK, Ntani G, Kontogiorgi M, Poulakou G, Armaganidis A, Dimopoulos G (2012) An ESICM systematic review and meta-analysis of procalcitonin-guided antibiotic therapy algorithms in adult critically ill patients. Intensive Care Med 38:940-949

25. Derde LP, Dautzenberg MJ, Bonten MJ (2012) Chlorhexidine body washing to control antimicrobial-resistant bacteria in intensive care units: a systematic review. Intensive Care Med 38:931-939

26. Silvestri L, de la Cal MA, van Saene HK (2012) Selective decontamination of the digestive tract: the mechanism of action is control of gut overgrowth. Intensive Care Med 38:1738-1750

27. Backes Y, van der Sluijs KF, Mackie DP, Tacke F, Koch A, Tenhunen JJ, Schultz MJ (2012) Usefulness of suPAR as a biological marker in patients with systemic inflammation or infection: a systematic review. Intensive Care Med 38:1418-1428

28. Dunser MW, Festic E, Dondorp A, Kissoon N, Ganbat T, Kwizera A, Haniffa R, Baker T, Schultz MJ (2012) Recommendations for sepsis management in resource-limited settings. Intensive Care Med 38:557-574

29. Eisen DP (2012) Manifold beneficial effects of acetyl salicylic acid and nonsteroidal anti-inflammatory drugs on sepsis. Intensive Care Med 38:1249-1257

30. Hicks CW, Sweeney DA, Cui X, Li Y, Eichacker PQ (2012) An overview of anthrax infection including the recently identified form of disease in injection drug users. Intensive Care Med 38:1092-1104

31. Massion PB, Peters P, Ledoux D, Zimermann V, Canivet JL, Massion PP, Damas P, Gothot A (2012) Persistent hypocoagulability in patients with septic shock predicts greater hospital mortality: impact of impaired thrombin generation. Intensive Care Med 38:1326-1335

32. Heilmann C, Geisen U, Beyersdorf F, Nakamura L, Benk C, Trummer G, Berchtold-Herz M, Schlensak C, Zieger B (2012) Acquired von Willebrand syndrome in patients with extracorporeal life support (ECLS). Intensive Care Med 38:62-68 
33. Schaden E, Metnitz PG, Pfanner G, Heil S, Pernerstorfer T, Perger P, Schoechl H, Fries D, Guetl M, KozekLangenecker S (2012) Coagulation Day 2010: an Austrian survey on the routine of thromboprophylaxis in intensive care. Intensive Care Med 38:984-990

34. Cheng SS, Nordenholz K, Matero D, Pearlman N, McCarter M, Gajdos C, Hamiel C, Baer A, Luzier E, Tran ZV, Olson T, Queensland K, Lutz R, Wischmeyer P (2012) Standard subcutaneous dosing of unfractionated heparin for venous thromboembolism prophylaxis in surgical ICU patients leads to subtherapeutic factor $\mathrm{Xa}$ inhibition. Intensive Care Med 38:642-648

35. Dellinger RP, Levy MM, Carlet JM, Bion J, Parker MM, Jaeschke R, Reinhart K, Angus DC, Brun-Buisson C, Beale R, Calandra T, Dhainaut JF, Gerlach H, Harvey M, Marini JJ, Marshall J, Ranieri M, Ramsay G, Sevransky J, Thompson BT, Townsend S, Vender JS, Zimmerman JL, Vincent JL (2008) Surviving Sepsis Campaign: international guidelines for management of severe sepsis and septic shock: 2008. Intensive Care Med 34:17-60

36. Rivers E, Nguyen B, Havstad S, Ressler J, Muzzin A, Knoblich B, Peterson E, Tomlanovich M (2001) Early goaldirected therapy in the treatment of severe sepsis and septic shock. N Engl J Med 345:1368-1377

37. Jones AE, Shapiro NI, Trzeciak S, Arnold RC, Claremont HA, Kline JA, Emergency Medicine Shock Research Network Investigators (2010) Lactate clearance vs central venous oxygen saturation as goals of early sepsis therapy: a randomized clinical trial. JAMA 303:739-746

38. De Backer D, Ospina-Tascon G, Salgado D, Favory R, Creteur J, Vincent JL (2010) Monitoring the microcirculation in the critically ill patient: current methods and future approaches. Intensive Care Med 36:1813-1825

39. Myers DE, Anderson LD, Seifert RP, Ortner JP, Cooper CE, Beilman GJ, Mowlem JD (2005) Noninvasive method for measuring local hemoglobin oxygen saturation in tissue using wide gap second derivative near-infrared spectroscopy. J Biomed Opt 10:034017

40. Payen D, Luengo C, Heyer L, RescheRigon M, Kerever S, Damoisel C, Losser MR (2009) Is thenar tissue hemoglobin oxygen saturation in septic shock related to macrohemodynamic variables and outcome? Crit Care 13(Suppl 5):S6
41. Creteur J, Carollo T, Soldati G, Buchele G, De Backer D, Vincent JL (2007) The prognostic value of muscle $\mathrm{StO}_{2}$ in septic patients. Intensive Care Med 33:1549-1556

42. Georger JF, Hamzaoui O, Chaari A, Maizel J, Richard C, Teboul JL (2010) Restoring arterial pressure with norepinephrine improves muscle tissue oxygenation assessed by near-infrared spectroscopy in severely hypotensive septic patients. Intensive Care Med 36:1882-1889

43. Mesquida J, Espinal C, Gruartmoner G, Masip J, Sabatier C, Baigorri F, Pinsky MR, Artigas A (2012) Prognostic implications of tissue oxygen saturation in human septic shock. Intensive Care Med 38:592-597

44. Lima A, van Genderen ME, Klijn E, Bakker J, van Bommel J (2012) Peripheral vasoconstriction influences thenar oxygen saturation as measured by near-infrared spectroscopy. Intensive Care Med 38:606-611

45. Lima A, Jansen TC, van Bommel J, Ince C, Bakker J (2009) The prognostic value of the subjective assessment of peripheral perfusion in critically ill patients. Crit Care Med 37:934-938

46. Kaplan LJ, McPartland K, Santora TA, Trooskin SZ (2001) Start with a subjective assessment of skin temperature to identify hypoperfusion in intensive care unit patients. J Trauma 50:620-627 (discussion 627-628)

47. Bartels SA, Bezemer R, de Vries FJ, Milstein DM, Lima A, Cherpanath TG, van den Meiracker AH, van Bommel J, Heger M, Karemaker JM, Ince C (2011) Multi-site and multi-depth near-infrared spectroscopy in a model of simulated (central) hypovolemia: lower body negative pressure. Intensive Care Med 37:671-677

48. Ait-Oufella H, Joffre J, Boelle PY, Galbois A, Bourcier S, Baudel JL, Margetis D, Alves M, Offenstadt G, Guidet B, Maury E (2012) Knee area tissue oxygen saturation is predictive of 14-day mortality in septic shock. Intensive Care Med 38:976-983

49. Brown KA, Brain SD, Pearson JD, Edgeworth JD, Lewis SM, Treacher DF (2006) Neutrophils in development of multiple organ failure in sepsis. Lancet 368:157-169

50. Buras JA, Reenstra WR (2007) Endothelial-neutrophil interactions during ischemia and reperfusion injury: basic mechanisms of hyperbaric oxygen. Neurol Res 29:127-131

51. Bertuglia S, Colantuoni A (2000) Protective effects of leukopenia and tissue plasminogen activator in microvascular ischemia-reperfusion injury. Am J Physiol Heart Circ Physiol 278:H755-H761
52. Karvunidis T, Chvojka J, Lysak D, Sykora R, Krouzecky A, Radej J, Novak I, Matejovic M (2012) Septic shock and chemotherapy-induced cytopenia: effects on microcirculation. Intensive Care Med 38:1336-1344

53. Guyton AC (1955) Determination of cardiac output by equating venous return curves with cardiac response curves. Physiol Rev 35:123-129

54. Maas JJ, Pinsky MR, Geerts BF, de Wilde RB, Jansen JR (2012) Estimation of mean systemic filling pressure in postoperative cardiac surgery patients with three methods. Intensive Care Med 38:1452-1460

55. Maas JJ, Geerts BF, van den Berg PC, Pinsky MR, Jansen JR (2009) Assessment of venous return curve and mean systemic filling pressure in postoperative cardiac surgery patients. Crit Care Med 37:912-918

56. Pirracchio R, Cholley B, De Hert S, Solal AC, Mebazaa A (2007) Diastolic heart failure in anaesthesia and critical care. Br J Anaesth 98:707-721

57. Mahjoub Y, Benoit-Fallet H, Airapetian N, Lorne E, Levrard M, Seydi AA, Amennouche N, Slama M, Dupont H (2012) Improvement of left ventricular relaxation as assessed by tissue Doppler imaging in fluid-responsive critically ill septic patients. Intensive Care Med 38:1461-1470

58. Saugel B, Fassio F, Hapfelmeier A, Meidert AS, Schmid RM, Huber W (2012) The T-line TL-200 system for continuous non-invasive blood pressure measurement in medical intensive care unit patients. Intensive Care Med 38:1471-1477

59. Pierrakos C, Velissaris D, Scolletta S, Heenen S, De Backer D, Vincent JL (2012) Can changes in arterial pressure be used to detect changes in cardiac index during fluid challenge in patients with septic shock? Intensive Care Med 38:422-428

60. Leatherman JW, Bastin-Dejong C, Shapiro RS, Saavedra-Romero R (2012) Use of expiratory change in bladder pressure to assess expiratory muscle activity in patients with large respiratory excursions in central venous pressure. Intensive Care Med 38:453-457

61. Hartog CS, Skupin H, Natanson C, Sun J, Reinhart K (2012) Systematic analysis of hydroxyethyl starch (HES) reviews: proliferation of low-quality reviews overwhelms the results of wellperformed meta-analyses. Intensive Care Med 38:1258-1271 
62. Thomas-Rueddel DO, Vlasakov V, Reinhart K, Jaeschke R, Rueddel H, Hutagalung R, Stacke A, Hartog CS (2012) Safety of gelatin for volume resuscitation-a systematic review and meta-analysis. Intensive Care Med 38:1134-1142

63. Sandroni C, Cavallaro F, Marano C, Falcone C, De Santis P, Antonelli M (2012) Accuracy of plethysmographic indices as predictors of fluid responsiveness in mechanically ventilated adults: a systematic review and meta-analysis. Intensive Care Med 38:1429-1437

64. Tacon CL, McCaffrey J, Delaney A (2012) Dobutamine for patients with severe heart failure: a systematic review and meta-analysis of randomised controlled trials. Intensive Care Med 38:359-367

65. Polito A, Parisini E, Ricci Z, Picardo S, Annane D (2012) Vasopressin for treatment of vasodilatory shock: an ESICM systematic review and metaanalysis. Intensive Care Med 38:9-19

66. Schmittinger CA, Torgersen C, Luckner G, Schroder DC, Lorenz I, Dunser MW (2012) Adverse cardiac events during catecholamine vasopressor therapy: a prospective observational study. Intensive Care Med 38:950-958

67. Balzer F, Wickboldt N, Spies C, Walder B, Goncerut J, Citerio G, Rhodes A, Kastrup M, Boemke W (2012) Standardised drug labelling in intensive care: results of an international survey among ESICM members. Intensive Care Med 38:1298-1305

68. Rhodes A, Ferdinande P, Flaatten H, Guidet B, Metnitz PG, Moreno RP (2012) The variability of critical care bed numbers in Europe. Intensive Care Med 38:1647-1653

69. Kanhere MH, Kanhere HA, Cameron A, Maddern GJ (2012) Does patient volume affect clinical outcomes in adult intensive care units? Intensive Care Med 38:741-751

70. Quenot JP, Rigaud JP, Prin S, Barbar S, Pavon A, Hamet M, Jacquiot N, Blettery B, Herve C, Charles PE, Moutel G (2012) Suffering among carers working in critical care can be reduced by an intensive communication strategy on end-of-life practices. Intensive Care Med 38:55-61

71. Fuchs L, Chronaki CE, Park S, Novack V, Baumfeld Y, Scott D, McLennan S, Talmor D, Celi L (2012) ICU admission characteristics and mortality rates among elderly and very elderly patients. Intensive Care Med 38:1654-1661
72. Song JU, Suh GY, Park HY, Lim SY, Han SG, Kang YR, Kwon OJ, Woo S, Jeon K (2012) Early intervention on the outcomes in critically ill cancer patients admitted to intensive care units. Intensive Care Med 38:1505-1513

73. Gillies MA, McKenzie CA, Whiteley C, Beale RJ, Tibby SM (2012) Safety of nicotine replacement therapy in critically ill smokers: a retrospective cohort study. Intensive Care Med 38:1683-1688

74. Mosquera VX, Marini M, Muniz J, Asorey-Veiga V, Adrio-Nazar B, Boix R, Lopez-Perez JM, Pradas-Montilla G, Cuenca JJ (2012) Traumatic aortic injury score (TRAINS): an easy and simple score for early detection of traumatic aortic injuries in major trauma patients with associated blunt chest trauma. Intensive Care Med 38:1487-1496

75. McKinley S, Aitken LM, Alison JA, King M, Leslie G, Burmeister E, Elliott D (2012) Sleep and other factors associated with mental health and psychological distress after intensive care for critical illness. Intensive Care Med 38:627-633

76. Volpicelli G, Elbarbary M, Blaivas M, Lichtenstein DA, Mathis G, Kirkpatrick AW, Melniker L, Gargani L, Noble VE, Via G, Dean A, Tsung JW, Soldati G, Copetti R, Bouhemad B, Reissig A, Agricola E, Rouby JJ, Arbelot C, Liteplo A, Sargsyan A, Silva F, Hoppmann R, Breitkreutz R, Seibel A, Neri L, Storti E, Petrovic T (2012) International evidence-based recommendations for point-of-care lung ultrasound. Intensive Care Med 38:577-591

77. Lamperti M, Bodenham AR, Pittiruti M, Blaivas M, Augoustides JG, Elbarbary M, Pirotte T, Karakitsos D, Ledonne J, Doniger S, Scoppettuolo G, Feller-Kopman D, Schummer W, Biffi R, Desruennes E, Melniker LA, Verghese ST (2012) International evidence-based recommendations on ultrasound-guided vascular access. Intensive Care Med 38:1105-1117

78. Schnell D, Darmon M (2012) Renal Doppler to assess renal perfusion in the critically ill: a reappraisal. Intensive Care Med 38:1751-1760

79. Lakhal K, Serveaux-Delous M, Lefrant JY, Capdevila X, Jaber S (2012) Chest radiographs in 104 French ICUs: current prescription strategies and clinical value (the RadioDay study). Intensive Care Med 38:1787-1799
80. Rohacek M, Buatsi J, Szucs-Farkas Z, Kleim B, Zimmermann H, Exadaktylos A, Stoupis C (2012) Ordering CT pulmonary angiography to exclude pulmonary embolism: defense versus evidence in the emergency room. Intensive Care Med 38:1345-1351

81. Barrett KA, Ferguson ND, Athaide V, Cook DJ, Friedrich JO, McDonald E, Pinto R, Smith OM, Stevenson J, Scales DC (2012) Surrogate decision makers' attitudes towards research decision making for critically ill patients. Intensive Care Med 38:1616-1623

82. Mehta S, Quittnat Pelletier F, Brown M, Ethier C, Wells D, Burry L, MacDonald R (2012) Why substitute decision makers provide or decline consent for ICU research studies: a questionnaire study. Intensive Care Med 38:47-54

83. Schenker Y, Tiver GA, Hong SY, White DB (2012) Association between physicians' beliefs and the option of comfort care for critically ill patients. Intensive Care Med 38:1607-1615

84. Forte DN, Vincent JL, Velasco IT, Park M (2012) Association between education in EOL care and variability in EOL practice: a survey of ICU physicians. Intensive Care Med 38:404-412

85. Barnato AE, Tate JA, Rodriguez KL, Zickmund SL, Arnold RM (2012) Norms of decision making in the ICU: a case study of two academic medical centers at the extremes of end-of-life treatment intensity. Intensive Care Med 38:1886-1896

86. Azoulay E, Garrouste M, GoldgranToledano D, Adrie C, Max A, Vesin A, Francais A, Zahar JR, Cohen Y, Allaouchiche B, Schlemmer B, Timsit JF (2012) Increased nonbeneficial care in patients spending their birthday in the ICU. Intensive Care Med 38:1169-1176

87. Bulow HH, Sprung CL, Baras M, Carmel S, Svantesson M, Benbenishty J, Maia PA, Beishuizen A, Cohen S, Nalos D (2012) Are religion and religiosity important to end-of-life decisions and patient autonomy in the ICU? The Ethicatt study. Intensive Care Med 38:1126-1133

88. Quenot JP, Rigaud JP, Prin S, Barbar S, Pavon A, Hamet M, Jacquiot N, Blettery B, Herve C, Charles PE, Moutel G (2012) Impact of an intensive communication strategy on end-of-life practices in the intensive care unit. Intensive Care Med 38:145-152 
89. Ouanes I, Stambouli N, Dachraoui F, Ouanes-Besbes L, Toumi S, Ben Salem F, Gahbiche M, Abroug F (2012)

Pattern of end-of-life decisions in two

Tunisian intensive care units: the role of culture and intensivists' training. Intensive Care Med 38:710-717

90. Louriz M, Abidi K, Akkaoui M, Madani N, Chater K, Belayachi J, Dendane T, Zeggwagh AA, Abouqal R (2012)

Determinants and outcomes associated with decisions to deny or to delay intensive care unit admission in

Morocco. Intensive Care Med 38:830-837
91. Gristina GR, Martin E, Ranieri VM (2012) Regulation of advance directives in Italy: a bad law in the making. Intensive Care Med 38:1897-1900

92. Bein T, Hackner K, Zou T, Schultes S, Bosch T, Schlitt HJ, Graf BM, Olden M, Leitzmann M (2012) Socioeconomic status, severity of disease and level of family members' care in adult surgical intensive care patients: the prospective ECSSTASI study. Intensive Care Med 38:612-619
93. Mealer M, Jones J, Moss M (2012) A qualitative study of resilience and posttraumatic stress disorder in United States ICU nurses. Intensive Care Med 38:1445-1451 